\title{
Serotonin receptor modulators in the treatment of irritable bowel syndrome
}

\author{
Mohammad Fayyaz \\ Jeffrey M Lackner \\ Division of Gastroenterology, \\ Department of Medicine, University \\ at Buffalo School of Medicine, SUNY, \\ Buffalo, NY, USA
}

\begin{abstract}
The aim of this article is to review the pathophysiology and clinical role of serotonin receptor modulators used in the treatment of irritable bowel syndrome. Serotonin is an important monoamine neurotransmitter that plays a key role in the initiation of peristaltic and secretory reflexes, and in modulation of visceral sensations. Several serotonin receptor subtypes have been characterized, of which 5HT3, 5HT4, and 5HT1b are the most important for GI function. 5HT4 agonists (eg, tegaserod) potentiate peristalsis initiated by 5HT1 receptor stimulation. 5HT4 agonists are therefore useful in constipation predominant form of IBS and in chronic constipation. 5HT3 antagonists (Alosetron and Cilansetron) prevent the activation of 5HT3 receptors on extrinsic afferent neurons and can decrease the visceral pain associated with IBS. These agents also retard small intestinal and colonic transit, and are therefore useful in diarrhea-predominant IBS. Tegaserod has been demonstrated in several randomized, placebo controlled trials to relieve global IBS symptoms as well as individual symptoms of abdominal discomfort, number of bowel movements and stool consistency. Several randomized, controlled trials have shown that alosetron relieves pain, improves bowel function, and provides global symptom improvement in women with diarrhea-predominant irritable bowel syndrome. However, ischemic colitis and severe complications of constipation have been major concerns leading to voluntary withdrawal of Alosetron from the market followed by remarketing with a comprehensive risk management program.
\end{abstract}

Keywords: serotonin, irritable bowel syndrome, tegaserod

\section{Introduction}

Irritable bowl syndrome (IBS) is a common, often debilitating, gastrointestinal disorder with a worldwide prevalence rate of 10\%-20\% (Camilleri and Choi 1997). IBS ranks as the most common medical diagnosis among gastroenterologists and the seventh-leading diagnosis among primary care physicians in the U.S. (Sandler et al 2002). IBS is costly in terms of medical treatments and diagnostic procedures (Levy et al 2001), time lost from work (Hahn et al 1999), and nonmonetary costs such as diminished quality of life (Lackner et al in press) and activity limitations (Whitehead et al 1996). Each year, the direct (diagnostic testing, physician charges, drugs, hospital costs) and indirect (absenteeism, reduced work efficiency, income loss, transportation, early retirement) costs of IBS in the US are pegged at $\$ 1.7-\$ 10$ billion and $\$ 19.2$ billion, respectively (Talley et al 1995; American Gastroenterological Association 2002).

The diagnosis of IBS is based on the symptom-based classification system known as the Rome criteria (Drossman et al 2000). To meet diagnostic criteria for IBS, Rome criteria require that in the preceding 12 months the patient must experience 12 weeks (need not be consecutive) of abdominal pain or discomfort with two out of three features:

- Relieved with defecation; and/or

- Onset associated with a change in frequency of stool; and/or

- Onset associated with a change in appearance of stool. 
IBS can be either diarrhea predominant (IBS-D), constipation predominant (IBS-C) or present with diarrhea alternating with constipation (IBS-A).

\section{Therapy}

The management of this disorder should be guided by he basic ethical principal of nonmaleficence (first, do no harm), the establishment of a sound physician-patient relationship, and the judicious use of pharmacologic agents to target the patient's primary symptoms. Conventional treatments for irritable bowel syndrome include use of bulking agents, antispasmodics agents, antidiarrheal agents and tricyclic antidepressants. While these treatments can, for some patients, be effective for isolated symptoms, they have a disappointing track record for the full range of symptoms of IBS. This has fuelled intense research to develop newer medications for the treatment of this common disorder. The most exciting developments have been related to understanding the functioning of the enteric nervous system at the molecular level. Better understanding of the role of serotonin and serotonin receptors in intestinal motility as well as in gut-brain signaling has lead to development of newer drugs that that have been studied in several randomized controlled trials. These serotonergic agents have been demonstrated to have statistically significant but modest efficacy in patients with irritable bowel syndrome. There is hope that further research in this field will lead to development of medications that will be safe and effective, and will target specific disturbances in intestinal motility and visceral hypersensitivity at the molecular level.

\section{Enteric nervous system, serotonin and serotonin receptors}

Serotonin is a monoamine neurotransmitter of profound importance in the enteric nervous system. About $95 \%$ of the serotonin in the body is found in the GI tract; $90 \%$ is in enterochromaffin cells (EC cells) and the remaining $10 \%$ in enteric neurons. It plays a key role in the initiation of peristaltic and secretory reflexes (Grider et al 96), and in modulation of visceral sensations (Kilkens et al 2004). There are more than 25 receptor subtypes; 5HT3, 5HT4, and $5 \mathrm{HT} 1 \mathrm{~b}$ are most important for GI function. In the central nervous system, the highest levels of 5-HT3 receptor are in the brainstem, particularly in the nucleus tractus solitarius, area postrema, and dorsal motor nucleus of the vagus nerve (Gershon 2004; Baker 2005).

The function of the ENS is coordinated by the central nervous system. Parasympathetic efferent pathways consist of the vagus and sacral nerves. They exert an excitatory effect on the enteric neurons. The sympathetic efferent fibers inhibit GI activity. Sensory information is transmitted to the CNS via the vagus and splanchnic afferents. The splanchnic afferent neurons transmit pain sensation, whereas the parasympathetic afferent fibers transmit physiological information and nonnoxious sensation (Goyal and Hirano 1996; Baker 2005). The enteric nervous system is composed of two major interconnected plexuses of ganglia and nerve processes; the myenteric plexus and the submucous plexus. The myenteric plexus is located between the longitudinal and circular muscle layers whereas the submucous plexus is located in the submucosa between the muscularis mucosa and circular muscle layer (Goyal and Hirano 1996; Talley 2001; Baker 2005; Gershon 2005). Intrinsic primary afferent neurons (IPANs) have cell bodies in the myenteric plexus (myenteric IPANs) or in the submocus plexus (submucous IPANs) and processes extending to muscularis mucosa as well as processes synapsing with interneurons. IPANs are the primary sensors and regulators of the ENS. Interneurons involved in motor reflexes are either directed cephalad or caudally and are called ascending and descending interneurons respectively. Interneurons synapse with motor neurons that are either excitatory or inhibitory (Goyal and Hirano 1996; Gershon 2005). Excitatory motor neurons secrete Ach and Substance P whereas inhibitory motor neurons secrete NO, VIP, ATP and PACAP. EC cells are neuroendocrine cells that serve as sensory tranducers and transmit sensory information from the gut mucosa to the nerve fibers in the lamina propria. This communication takes place via the secretion of serotonin by EC cells into the lamina propria in response to luminal stimuli (Gershon 2005). The signal is terminated by the re-uptake of serotonin by the serotonin reuptake transporter (SERT) present on the enterocytes. Serotonin stimulates IPANs and information is thus transmitted from EC cells via IPANs to interneurons and motor or secretomotor neurons. Submucosal IPANs secrete acetylcholine (Ach) and calcitonin gene related peptide (CGRP) (Gershon 2005).

The peristaltic reflex can be elicited by stretch of the circular muscle layer by a bolus of food or by mucosal stimulation. Muscle stretch activates extrinsic sensory neurons with cell bodies in the dorsal root ganglion and axonal projections to the neurons of the ENS. This results in the release of CGRP by the enteric neurons without involvement of the EC cells or the release of serotonin. Mucosal stimulation by a bolus, on the other hand, results in the release of serotonin from the EC cells. Serotonin acts on the 5HT1p receptors on the terminals of submucosal IPANs resulting in the secretion of Ach and CGRP by the IPANs. 5HT4 receptors are present 
pre-synaptically on the distal terminals of submucosal IPANs. Stimulation of 5HT4 receptors by serotonin augments the release of Ach and CGRP. Both sensory pathways are coupled to the same effector limbs. Stimulation of 5HT4 receptors on submucosal IPANs by serotonin augments the release of Ach and CGRP. The released Ach and CGRP excite the ascending and descending interneurons, which in turn release Ach at their terminals. Presynaptic 5HT4 receptors also augment this Ach release (Grider 1996; Gershon 2004, 2005). The ascending interneurons synapse with excitatory motor neurons cephalad to the site of stimulus (bolus), which release Ach and substance P. This results in smooth muscle contraction cephalad to the bolus. The descending interneurons synapse with inhibitory motor neurons caudad to the bolus, which release NO and VIP, resulting in smooth muscle relaxation. This whole process results in propagation of the bolus in a coordinated fashion. 5HT1p receptors play a key role in the initiation of peristalsis, whereas 5HT4 receptors play a role in augmenting the release of neurotransmitters once the process is initiated by $5 \mathrm{HT} 1 \mathrm{p}$ receptor stimulation (Grider 1996; Gershon 2004, 2005). 5HT3 receptors are found on the terminals of extrinsic afferent neurons, and are involved in the transmission of sensory stimuli to the CNS. Noxious stimuli are carried by spinal afferents while nausea, bloating and other non-painful sensations are transmitted by parasympathetic afferents. 5HT3 receptors are also present on myenteric IPANs and on other neurons in the myenteric plexus where they mediate fast excitatory neurotransmission (Gershon 2004, 2005). 5HT3 receptors do not play a significant role in peristaltic and secretory reflexes, but potent antagonists can result in slowing of GI motility.

Deletion of SERT gene in mice results in diarrhea from excess serotonin availability, followed by constipation from desensitization of serotonin receptors (Chen et al 2001). The 5HT1p agonist 5-hydroxyindalapine causes severe diarrhea by activating submucosal IPANs (Gershon 2005). Similarly 5HTP-DP (N-acetyl-5-hydroxytryptophy-5-hydroxytryptophan amide) an antagonist of 5HT1p blocks peristaltic and secretory reflexes, and can cause paralytic ileus (Gershon 2005). The 5HT4 agonist tegaserod potentiates peristalsis initiated by 5 HT 1 receptor stimulation by acting on pre-synaptic 5HT4 receptors and augmenting the release of neurotransmitters (Grider 1996; Gershon 2004, 2005). 5HT4 agonists are therefore useful in constipation predominant form of IBS and in chronic constipation. 5HT4 receptors are not present on extrinsic afferent neurons and therefore do not directly modulate sensation. However, by improving bowel motility, they may improve abdominal pain. 5HT3 antagonists
(Alosetron and Cilansetron) prevent the activation of 5HT3 receptors on extrinsic afferent neurons and can decrease the visceral pain associated with IBS (Gershon 2005). They also decrease gastrointestinal motility, and are therefore useful in IBS-D.

Role of serotonin in the pathogenesis of IBS is being investigated. In a recent study (Atkinson et al 2005), 84 IBS patients and 35 healthy volunteers had platelet-depleted plasma 5-HT/5-HIAA concentrations measured before and after a standard meal. D-IBS patients had raised concentrations of 5-HT in their plasma probably as a consequence of reduced metabolism and uptake. However a 5-HT response to meal ingestion in these patients was demonstrated to be similar to healthy controls. On the other hand, c-IBS had a very limited 5-HT response to meal ingestion as demonstrated by the low concentrations of both 5-HT and 5-HIAA. These observations lead the authors to conclude that D- and C-IBS may be primarily disorders of reduced 5-HT reuptake and release, respectively. These are very interesting findings, but other studies are needed to replicate and validate these results.

\section{Tegaserod}

Tegaserod, an aminoguanidine indole derivative of serotonin, is a selective partial agonist of pre-synaptic 5HT4 receptors on submucosal IPANs (Camilleri 2001, 2002, 2004; Talley 2003; Chey 2004). It augments 5HT1 receptor mediated release of Ach and CGRP from submucosal IPANs, thereby potentiating the peristaltic reflex (Grider 1996; Gershon 2004, 2005). Tegaserod has been demonstrated to accelerate gastric emptying and small intestinal transit, and induce a small but significant acceleration of colonic transit during acute administration (Degan et al 2001). It accelerates orocecal transit in irritable bowel syndrome patients with constipation (Prather et al 2000). It has also been demonstrated to reduce the sensitivity to rectal distension in healthy subjects without altering the intensity of subjective pain perception or rectal compliance (Coffin et al 2003).

The efficacy and safety of tegaserod has been demonstrated by several large randomized controlled trials. In a randomized, placebo-controlled, double-blind, multicenter study of eight hundred and eighty-one patients with irritable bowel syndrome, tegaserod $2 \mathrm{mg}$ twice daily or $6 \mathrm{mg}$ twice daily showed a statistically significant relief of overall irritable bowel syndrome symptoms, measured by a weekly, self-administered questionnaire. There was also a statistically significant improvement in individual irritable bowel 
syndrome symptoms of abdominal discomfort/pain, number of bowel movements and stool consistency. Tegaserod was well tolerated, transient diarrhea being the only adverse event seen more frequently with tegaserod than placebo (MullerLissner et al 2001). In another randomized, double-blind, multicenter study of 1519 women tegaserod $6 \mathrm{mg}$ BID for 12 weeks produced significant improvements in the Subject's Global Assessment of Relief compared to placebo. These improvements were seen within the first week, and were maintained throughout the treatment period. However, at the end of the study period there was a smaller treatment difference $\mathrm{v} / \mathrm{s}$ placebo due to an increase in placebo response to $61 \%$ as compared to $67 \%$ in the treatment group. Diarrhea was the most frequent side effect but it lead to discontinuation of tegaserod in only $1.6 \%$ of patients (Novick et al 2002). Satisfactory relief from IBS symptoms was also demonstrated in a trial of 520 patients from the Asia-Pacific region with IBS, excluding those with diarrhea predominant IBS who were randomized to receive either tegaserod $6 \mathrm{mg}$ twice daily or placebo for a 12 week treatment period. A clinically relevant effect was observed as early as week 1 and was maintained throughout the treatment period (Kellow et al 2003). Similarly in an 8-week, open-label, prospective, multicenter study from Switzerland, tegaserod $6 \mathrm{mg}$ bid was demonstrated to be safe, effective, and well tolerated in the treatment of patients with non-diarrhea irritable bowel syndrome (Fried et al 2005). In a randomized, placebo controlled trial from Sweden, the odds of satisfactory relief from irritable bowel symptoms were $54 \%$ higher over weeks I -4 and $78 \%$ higher over weeks $1-12$ in the tegaserod (6 mg twice daily) group than in the placebo group. Diarrhea led to discontinuation in $2.8 \%$ of tegaserod patients (Nyhlin 2004). In a meta-analysis of randomized controlled trials, tegaserod showed a significant effect in women with an odds ratio of 1.4 (CI: 1.2-1.5) (Lesbros-Pantoflickova et al 2004). A systematic review of eight trials (IBS) showed relative risk (RR) of being a responder in terms of global relief of GI symptoms was significantly higher with tegaserod $12 \mathrm{mg}$ (number needed to treat of 14) and tegaserod $4 \mathrm{mg}$ (number needed to treat of 20) compared with placebo. When all tegaserod doses were combined the $\mathrm{RR}$ of being a responder was $1.17(95 \%$ CI 1.08, 1.27), with a number needed to treat of 17. Symptoms indicative of GI motility such as number of bowel movements and days without bowel movements were improved consistently. Tegaserod did not significantly improve abdominal pain and discomfort. (Evans et al 2004). In a randomized trial (Kkoshoo et al 2006), 48 patients with constipation predominant irritable bowel syndrome were allocated to treatment with a laxative alone (polyethylene glycol 3350 oral solution) or combination therapy with the laxative and tegaserod. Treatment with the laxative alone resulted in significant increase in frequency of bowel movements but not significant improvement in pain. The combination therapy led to significant increase in the frequency of bowel movements and also significant reduction in pain. Re-treatment with tegaserod for recurrence of symptoms after an eight week withdrawal period has been demonstrated to be as efficacious and safe as the initial treatment (Muller-Lissner et al 2005). Tegaserod has been shown to significantly reduce work productivity loss by 2.5 hours per week, calculated on the basis of a 40 hour work week (Reilly et al 2005). In a model based on economic and epidemiologic published literature and clinical trial results, medical therapy for IBS with tegaserod was predicted to be cost-effective from an employer's perspective for the treatment of women with IBS-C (Smith et al 2005).

Tegaserod may also be useful in conditions other than IBS, like chronic constipation and non-ulcer dyspepsia. In a randomized trial of 1264 patients with chronic constipation there was a significant increase in complete spontaneous bowel movements per week in patients receiving tegaserod. The number needed to treat was 7.3 for the $6 \mathrm{mg}$ twice daily dose compared with 11.1 for tegaserod $2 \mathrm{mg}$ twice daily. Significant improvements were also seen in stool form and in global assessment of bowel habits and constipation. The most common adverse events, headache and abdominal pain, were more frequent with placebo than with tegaserod (Johanson 2004; Kamm et al 2005). However, head to head comparison with more conventional laxatives is needed before its use can be justified for this indication. Tegaserod may a have a role in the management of some patients with non ulcer dyspepsia (Cremonini et al 2004). Tegaserod has been demonstrated to accelerate the return of gastric function in intensive care unit patients with gastroparesis in case reports (Bank et al 2005). The most common adverse effect is diarrhea. In randomized controlled trials diarrhea was reported by $8.8 \%$ of patients treated with tegaserod versus $3.8 \%$ of patients receiving placebo (Schoenfeld 2004).

As far as the safety is concerned, no cases of ischemic colitis were reported among 7000 patients randomly assigned to receive tegaserod in placebo-controlled trials of at least three months' duration. However, between August 2002 and March 2004, the FDA received 20 reports of cases of ischemic colitis associated with the use of tegaserod. As a result of these reports, the labeling of tegaserod (Zelnorm) was updated to alert the prescribers of the risk of 
tegaserod-associated ischemic colitis (Brinker et al 2004; DiBaise 2005). Higher cholecystectomy rates have been noted among tegaserod treated but no causal relationship has been established. Higher rates of cholecystectomy, appendectomy and hysterectomy have also been reported in IBS patients in general unrelated to any medication use. Gallbladder contractility variables were similar after $2 \mathrm{wk}$ of treatment with tegaserod $6 \mathrm{mg}$ twice daily and placebo in healthy female subjects and female patients with IBS-C. There were no significant changes in the luminal diameters of the common hepatic duct or common bile duct after tegaserod compared to placebo, and no significant ductal dilation was observed during maximal gallbladder emptying (Fisher et al 1999). Tegaserod-treated patients in RCTs have not demonstrated significant prolongation of the QTc interval or cardiac arrhythmias compared with placebo-treated patients. Supra-therapeutic doses in healthy volunteers did not affect electrocardiographic parameters (Morganroth et al 2002). No clinically significant adverse drug-drug interactions have been reported. Manufacturer listed contraindications include severe renal impairment, moderate or severe hepatic impairment, history of bowel obstruction, symptomatic gallbladder disease, suspected sphincter of Oddi dysfunction, or abdominal adhesions. Treatment should not be started in patients with diarrhea or in those who experience diarrhea frequently. Pregnancy category is $\mathrm{B}$, and it not recommended during lactation. In February and March 2007, FDA reviewed the data of 29 studies that included 11,614 patients treated with tegaserod and 7,031 treated with placebo. The patients treated with tegaserod had a higher chance of having a myocardial infarction, stroke or angina than those who were treated with placebo $(0.1 \%$ vs $0.01 \%)$. A public health advisory was released by FDA in March 2007, and tegaserod was taken off the market by Novartis. In July 2007, FDA announced that it is permitting restricted use of tegaserod under an investigational new drug (IND) protocol to treat IBS-C and chronic idiopathic constipation in women younger than 55 who meet specific guidelines.

\section{Alosetron}

5HT3 receptors are present on the intrinsic neurons within the myenteric plexus and play a minor role in the ENS neurotransmission. Being a potent $5 \mathrm{HT} 3$ antagonist, alosetron can slow intestinal motility. More importantly, 5HT3 receptors are present on the extrinsic sensory neurons and vagal afferents involved in the transmission of noxious and non-noxious (eg, nausea, bloating) sensations respectively. A 5HT3 antagonist can be expected to inhibit these sensations (Gershon 2005).
Alosetron significantly retards small intestinal (Veramontes et al 2001) and colonic (Houghton et al 2000; Veramontes et al 2001) transit in diarrhea-predominant IBS patients; this effect is more prominent in females (Veramontes et al 2001). Alosetron has been demonstrated, on isobaric phasic distension studies, to increases the compliance of the colon to distension. This was postulated to contribute to changes in perception of colonic distension (Delvaux et al 1998). Alosetron increases the number and propagation length of high amplitude colonic contractions which results in a paradoxical decrease in stool frequency (Clemens et al 2002). Noxious intestinal stimuli like colorectal distension elicit a drop in the blood pressure in rats. This reflex depressor response can be used as a measure of visceral nociception. Alosetron has been shown to inhibit this vasodepressor response to colorectal distension by blocking the 5HT3 receptors (Kozlowski et al 2000). In functional brain imaging studies, alosetron has been shown to decrease brain activity in bilateral frontotemporal cortex and various limbic structures in response to colorectal distension (Mayer et al 2002).

Several multicenter, randomized, placebo controlled trials (Lembo et al 1996; Jones et al 1999; Camilleri et al 2000, 2001; Watson et al 2001; Wolfe at al 2001) have shown that alosetron relieves pain, improves bowel function, controls bowel urgency, provides global symptom improvement and improves quality of life in women with diarrhea-predominant irritable bowel syndrome. In a randomized controlled trial of 714 patients (Chey et al 2004) alosetron-treated patients had significantly greater 48 -wk average adequate relief $(p=0.01)$ and urgency control $(\mathrm{p}<0.001)$ rates compared with placebo. Adverse events were similar between treatment groups, except for constipation. Neither ischemic colitis nor serious events related to bowel motor dysfunction was reported.

In a meta-analysis of six high quality randomized controlled trials (Cremonini et al 2003) the odds ratio for adequate relief of pain or global symptoms improvement with alosetron $1 \mathrm{mg}$ twice a day was 1.81 (95\% confidence interval 1.57-2.10). The number needed to treat to achieve improvement in one patient over placebo was seven. 93\% of the patients were females, and 75\% had diarrhea predominant IBS. In another meta-analysis of randomized controlled trials (Lesbros-Pantoflickova et al 2004) alosetron was found to be effective in treatment of women with diarrhea predominant IBS with OR of 2.2 (95\% CI: 1.9-2.6).

Safety issues have overshadowed the reputation of alosetron. 18 cases of ischemic colitis and 11 cases of serious complications of constipation were reported out of 11,874 patients taking alosetron during alosetron's clinical trials' 
experience. During post marketing surveillance, the Food and Drug Administration received reports of 84 cases of ischemic colitis (including two deaths) and 113 cases of serious complications of constipation (including two deaths) associated with the use of alosetron from March 2000 to March 2002 (Avigan et al 2003). GlaxoSmithKline voluntarily withdrew it from the market in November, 2000. In June 2002, the FDA approved the remarketing of alosetron with a comprehensive risk management program. In this program, the prescribers attest to their knowledge and abilities in managing IBS and to their knowledge of the potential complications of alosetron. In addition, the prescribers agree to educating the patients about alosetron's risks and benefits and reporting serious adverse events to GSK and the FDA. The drug is indicated only in women with severe diarrhea-predominant IBS who have chronic IBS symptoms, who experience failure of the usual therapies for IBS, and who have had other GI diseases ruled out. Another recommendation was to start at a reduced dose of $1 \mathrm{mg}$ once daily, which can be increased to $1 \mathrm{mg}$ twice daily after four weeks if the lower dose is well-tolerated but the symptoms are not adequately controlled. 37 serious adverse events have been reported since alosetron was reintroduced to the market (Traynor 2004), including 6 cases of ischemic colitis. No deaths attributable to alosetron have been reported since its reintroduction into the market. Alosetron is contraindicated in patients with constipation. However no risk factors have been identified that can predict the development of ischemic colitis in alosetron treated patients.

\section{Newer agents}

A number of newer serotonin receptor modulators are being developed in an attempt to reduce the side effects while maintaining efficacy. These include renzapride, cilansetron, and ramosetron among others. Renzapride is a benzamide derivative with combined $5-\mathrm{HT}_{3}$-receptor antagonist and $5-\mathrm{HT}_{4}$-receptor agonist activity. In a dose-ranging pharmacodynamic study, it caused clinically significant doserelated acceleration of colonic transit. This acceleration of transit was associated with improvement of bowel function in female IBS-C patients (Camilleri et al 2004). Phase III clinical trials are currently underway. Cilansetron is another $5-\mathrm{HT}_{3}$ antagonist that has been investigated for treatment of diarrhea-predominant IBS. It reduces diarrhea and pain but there have been reports of severe constipation and ischemic colitis (Bradette et al 2004; Coremans et al 2004). It did not received FDA approval because of these concerns. Ramosetron, a 5-HT 3 -receptor antagonist with antiemetic properties, is being investigated in phase II trials in Japan for treatment of diarrhea predominant IBS. Newer partial agonists of the 5-HT ${ }_{3}$ receptor like ME3412 (5-chloro-2-(1,4-diazacycloheptan-1-yl)-7-methylbenzoxazole), may relieve diarrhea with low risk of inducing constipation (Kawano et al 2005).

\section{Selective serotonin reuptake inhibitors}

SSRIs are typically used in IBS patients as antidepressants, especially in patients with co-existing depressive disorder, anxiety disorder, panic disorder, or phobias. Any potential effects on serotonergic neurotransmission at the level of enteric nervous system have not been well studied. Fluoxetine has been shown to decrease orocecal and whole gut transit times in both constipation-predominant IBS patients and controls (Gorard et al 1994). In another study (Kuiken et al 2003) forty non-depressed IBS patients underwent a rectal barostat study to assess the sensitivity to rectal distention before and after 6 weeks of treatment with fluoxetine $20 \mathrm{mg}$ or placebo. At baseline, 21 of 40 patients showed hypersensitivity to rectal distention. Fluoxetine did not significantly alter the threshold for discomfort/pain relative to placebo, either in hypersensitive or in normosensitive IBS patients. Overall there was no significant difference in abdominal pain scores at six weeks, but in the subgroup of hypersensitive patients, fluoxetine significantly reduced the number of patients reporting abdominal pain.

In a study of forty-four cases of irritable bowel syndrome with predominance of pain and constipation (Vahedi et al 2005), fluoxetine was significantly more effective than placebo in decreasing abdominal discomfort, relieving feeling of bloating, increasing frequency of bowel movements and decreasing consistency of stool. Mean number of symptoms per patient decreased from 4.6 to 0.7 in the fluoxetine group vs. 4.5 to 2.9 in controls ( $p<0.001$ ). In a trial of paroxitine versus placebo among patients already on a high fiber diet (Tabas et al 2004; Talley 2004), overall well-being improved more with paroxetine than with placebo (63.3\% vs $26.3 \%$; $\mathrm{p}=0.01$ ), but abdominal pain, bloating, and social functioning did not. This improvement in well being held true for non-depressed IBS patients as well. For patients with severe IBS, both psychotherapy and paroxetine have been shown to improve health-related quality of life at no additional cost (Creed et al 2003). More recently, Tack et al (2006) found that the SSRI citalapram improved the severity of IBS symptoms including pain in a placebo-controlled study of 23 non-depressed IBS patients. Changes in pain were independent of changes in anxiety or depression, suggesting that SSRI may have peripheral benefits. While promising, 
enthusiasm is tempered by the sample size was small and the lack of significant impact on stool pattern.

\section{Conclusion}

Serotonin plays a key role in the initiation of peristaltic and secretory reflexes, and in modulation of visceral sensations. 5HT4 agonists (eg, tegaserod) potentiate peristalsis initiated by $5 \mathrm{HT} 1$ receptor stimulation by acting on pre-synaptic $5 \mathrm{HT} 4$ receptors and augmenting the release of neurotransmitters. 5HT4 agonists are therefore useful in constipation predominant form of IBS and in chronic constipation. 5HT3 antagonists (Alosetron and Cilansetron) prevent the activation of 5HT3 receptors on extrinsic afferent neurons and can decrease the visceral pain associated with IBS. They decrease gastrointestinal motility, and are therefore useful in IBS-D. Serotonin receptor modulators are an area of active research, and there is hope that newer agents will be developed, that are more effective and better tolerated.

\section{Grant support}

Preparation of this paper was supported in part by National Institutes of Health Grants DK-54211 and DK- 67878.

\section{References}

American Gastroenterological Association. 2002. The burden of gastrointestinal diseases. AGA Press.

Atkinson W, Lockhart S, Whorwell PJ, et al. 2006. Altered 5-hydroxytryptamine signaling in patients with constipation- and diarrhea-predominant irritable bowel syndrome. Gastroenterology, 130:34-43.

Avigan M, Justice R, Mackey AC, et al. 2003. Re: Brandt et al. An evidencebased approach to the management of irritable bowel syndrome in North America. Am J Gastroenterol, 98:2105.

Baker DE. 2005. Rationale for using serotonergic agents to treat irritable bowel syndrome. Am J Health-syst Pharm, 62:700-13.

Banh HL, MacLean C, Topp T, et al. 2005. The use of tegaserod in critically ill patients with impaired gastric motility. Clinical Pharmacology and Therapeutics, 77:583-6.

Bradette M, Moennikes H, Carter F, et al. 2004. Cilansetron in irritable bowel syndrome with diarrhea predominance (IBS-D): efficacy and safety in a 6 month global study. Gastroenterology, 126:A42.

Brinker AD, Mackey AC, Prizont R. 2004. Tegaserod and ischemic colitis. New England Journal of Medicine, 351:1361-4.

Camilleri M, Choi MG. 1997. Review article: irritable bowel syndrome Aliment Pharmacol Ther, 11:3-15.

Camilleri M, Northcutt AR, Kong S, et al. 2000. Efficacy and safety of alosetron in women with irritable bowel syndrome: a randomised, placebo-controlled trial. Lancet, 355:1035-40.

Camilleri M. 2001. Review article: tegaserod. Alimentary Pharmacology and Therapeutics, 15:277-89.

Camilleri M, Chey WY, Mayer EA, et al. 2001. A randomized controlled clinical trial of the serotonin type 3 receptor antagonist alosetron in women with diarrhea-predominant irritable bowel syndrome. Archives of Internal Medicine, 161:1733-40.

Camilleri M, Heading RC, Thompson WG. 2002. Clinical perspectives, mechanisms, diagnosis and management of irritable bowel syndrome. Alimentary Pharmacology and Therapeutics, 16:1407-30.
Camilleri M. 2004. Treating irritable bowel syndrome: overview, perspective and future therapies. British Journal of Pharmacology, 141:1237-48.

Camilleri M, McKinzie S, Fox J, et al. 2004. Effect of renzapride on transit in constipation-predominant irritable bowel syndrome. Clinical Gastroenterology and Hepatology, 2:895-904.

Chen JJ, Li Z, Pan H, et al. 2001. Maintance of serotonin in the intestinal mucosa and ganglia of mice that lack the high-affinity serotonin transporter: abnormal intestinal motility and the expression of cation transporters. J Neurosci, 21:6348-61.

Chey WD, Chey WY, Heath AT, et al. 2004. Long-term safety and efficacy of alosetron in women with severe diarrhea-predominant irritable bowel syndrome. American Journal of Gastroenterology, 99:2195.

Chey WD. 2004. Review article: tegaserod - the global experience. Alimentary Pharmacology and Therapeutics, 20(Suppl 7):15-19.

Clemens CH, Samsom M, Van Berge, et al. 2002. Effect of alosetron on left colonic motility in non-constipated patients with irritable bowel syndrome and healthy volunteers. Alimentary Pharmacology and Therapeutics, 16:993-1002.

Coffin B, Farmachidi JP, Rueegg P, et al. 2003. Tegaserod, a 5-HT4 receptor partial agonist, decreases sensitivity to rectal distension in healthy subjects. Alimentary Pharmacology and Therapeutics, 17:577-85.

Coremans G, Clouse RE, Carter F, et al. 2004. Cilansetron, a novel 5-HT3 antagonist, demonstrated efficacy in males with irritable bowel syndrome with diarrhea predominance (IBS-D). Gastroenterology, 126(Suppl 2):A-643. Abstract.

Creed F, Fernandes L, Guthrie E, et al. 2003. North of England IBS Research Group. The cost-effectiveness of psychotherapy and paroxetine for severe irritable bowel syndrome. Gastroenterology, 124:303.

Cremonini F, Delgado-Aros S, Camilleri M. 2003. Efficacy of alosetron in irritable bowel syndrome: a meta-analysis of randomized controlled trials. Neurogastroenterology and Motility, 15:79-86.

Cremonini F, Delgado-Aros S, Talley NJ. 2004. Functional dyspepsia: drugs for new (and old) therapeutic targets. Best Practice and Research in Clinical Gastroenterology, 18:717-33.

Degen L, Matzinger D, Merz M, et al. 2001. Tegaserod, a 5-HT4 receptor partial agonist, accelerates gastric emptying and gastrointestinal transit in healthy male subjects. Aliment Pharmacol Ther, 15:1745-51.

Drossman DA, Corazziari E, Talley NJ, et al. 2000. Rome II. The functional gastrointestinal disorders. Diagnosis, pathophysiology and treatment: A multinational consensus. Degnon Associates.

Delvaux M, Louvel D, Mamet JP, et al. 1998. Effect of alosetron on responses to colonic distension in patients with irritable bowel syndrome. Alimentary Pharmacology and Therapeutics, 12:849-55.

DiBaise JK. 2005. Tegaserod-associated ischemic colitis. Pharmacotherapy, 25:620-5.

Drossman DA, Camilleri M, Mayer EA, et al. 2002. AGA technical review on irritable bowel syndrome. Gastroenterology, 123:2108-31.

Evans BW, Clark WK, Moore DJ, et al. 2004. Tegaserod for the treatment of irritable bowel syndrome. Cochrane Database of Systematic Reviews. (1):CD003960.

Fisher RS, Thistle J, Lembo A, et al. 1999. Tegaserod does not alter fasting or meal-induced biliary tract motility. American Journal of Gastroenterology, 99:1342-9.

Fried M, Beglinger C, Bobalj NG, et al. 2005. TegaSwiss Study Group. Tegaserod is safe, well tolerated and effective in the treatment of patients with non-diarrhoea irritable bowel syndrome. European Journal of Gastroenterology and Hepatology, 17:421-7.

Gershon MD. 2004. Review article: serotonin receptors and transporters roles in normal and abnormal gastrointestinal motility. Alimentary Pharmacology and Therapeutics. 20(Supp1 7):3-14.

Gershon MD. 2005. Nerves, reflexes, and the enteric nervous system. J Clin Gastroenterol, 39:S184-93.

Gorard DA, Libby GW, Farthing MJG. 1994. Influence of antidepressants on whole gut and orocaecal transit time in health and irritable bowel syndrome. Aliment Pharmacol Ther, 8:159-66. 
Goyal RK, Hirano I. 1996. Mechanisms of disease: the enteric nervous system. NEJM, 334:1106-15.

Grider JR, Kuemmerle JF, Jin JG. 1996. 5-HT released by mucosal stimuli initiates peristalsis by activating 5-HT4/5-HT1p receptors on sensory CGRP neurons. Am J Physiol, 270:G778-82.

Hahn BA, Yan S, Strassels S. 1999. Impact of irritable bowel syndrome on quality of life and resource use in the United States and United Kingdom. Digestion, 60:77-81.

Houghton LA, Foster JM, Whorwell PJ. 2000. Alosetron, a 5-HT3 receptor antagonist, delays colonic transit in patients with irritable bowel syndrome and healthy volunteers. Alimentary Pharmacology and Therapeutics, 14:775-82.

Irvine EJ. 2004. Review: Tegaserod prompts global relief of symptoms in irritable bowel syndrome. ACP Journal Club, 141:44

Ishiguchi T, Itoh H, Ichinose M. 2004. Gastrointestinal motility and the brain-gut axis. Dig Endosc, 15:81-6.

Johanson JF. 2004. Review article: tegaserod for chronic constipation Alimentary Pharmacology and Therapeutics, 20(Suppl 7):20-4.

Jones RH, Holtmann G, Rodrigo L, et al. 1999. Alosetron relieves pain and improves bowel function compared with mebeverine in female nonconstipated irritable bowel syndrome patients. Alimentary Pharmacology and Therapeutics, 13:1419-27.

Kamm MA, Muller-Lissner S, Talley NJ, et al. 2005. Tegaserod for the treatment of chronic constipation: a randomized, double-blind, placebo-controlled multinational study. American Journal of Gastroenterology, 100:362-72.

Kawano K, Mori T, Fu L, et al. 2005. Comparison between partial agonist (ME3412) and antagonist (alosetron) of 5-hydroxytryptamine 3 receptor on gastrointestinal function. Neurogastroenterology and Motility, 17:290-301

Kellow J, Lee OY, Chang FY, et al. 2003. An Asia-Pacific, double blind, placebo controlled, randomised study to evaluate the efficacy, safety, and tolerability of tegaserod in patients with irritable bowel syndrome. Gut, 52:671-6.

Khoshoo V, Armstead C, Landry L. 2006. Effect of a laxative with and without tegaserod in adolescents with constipation predominant irritable bowel syndrome. Alimentary Pharmacology and Therapeutics, 23:191-6.

Kilkens TOC, Honig A, Nieuwenhoven MAV, et al. 2004. Acute tryptophan depletion affects brain-gut responses in irritable bowel syndrome patients and controls.

Kozlowski CM, Green A, Grundy D, et al. 2000. The 5-HT(3) receptor antagonist alosetron inhibits the colorectal distention induced depressor response and spinal c-fos expression in the anaesthetised rat. Gut, 46:474-80.

Kuiken SD, Tytgat GN, Boeckxstaens GE. 2003. The selective serotonin reuptake inhibitor fluoxetine does not change rectal sensitivity and symptoms in patients with irritable bowel syndrome: a double blind, randomized, placebo-controlled study. Clinical Gastroenterology and Hepatology, 1:219-28.

Lackner JM, Gudleski GD, Zack MM, et al. 2006. Measuring health-related quality of life in patients with irritable bowel syndrome: Can less be more? Psychosomatic Medicine, 68:312-20.

Lembo T, Wright RA, Bagby B, et al. Lotronex Investigator Team. 2001. Alosetron controls bowel urgency and provides global symptom improvement in women with diarrhea-predominant irritable bowel syndrome. American Journal of Gastroenterology, 96:2662-70.

Lesbros-Pantoflickova D, Michetti P, Fried M, et al. 2004. Meta-analysis: The treatment of irritable bowel syndrome. Alimentary Pharmacology and Therapeutics, 20:1253-69.

Levy RL, Von Korff MR, Whitehead WE, et al. 2001. Costs of care for irritable bowel syndrome patients in a health maintenance organization. Am J Gastroenterol, 96:3122-9.

Mayer EA, Berman S, Derbyshire SW, et al. 2002. The effect of the 5-HT3 receptor antagonist, alosetron, on brain responses to visceral stimulation in irritable bowel syndrome patients. Alimentary Pharmacology and Therapeutics, 16:1357-66.
Morganroth J, Ruegg PC, Dunger-Baldauf C, et al. 2002. Tegaserod, a 5 -hydroxytryptamine type 4 receptor partial agonist, is devoid of electrocardiographic effects. American Journal of Gastroenterology, 97:2321-7.

Muller-Lissner SA, Fumagalli I, Bardhan KD, et al. 2001. Tegaserod, a 5-HT(4) receptor partial agonist, relieves symptoms in irritable bowel syndrome patients with abdominal pain, bloating and constipation. Alimentary Pharmacology and Therapeutics, 15:1655-66.

Muller-Lissner S, Holtmann G, Rueegg P, et al. 2005. Tegaserod is effective in the initial and retreatment of irritable bowel syndrome with constipation. Alimentary Pharmacology and Therapeutics, 21:11-20.

Novick J, Miner P, Krause R, et al. 2002. A randomized, double-blind, placebo-controlled trial of tegaserod in female patients suffering from irritable bowel syndrome with constipation. Alimentary Pharmacology and Therapeutics, 16:1877-88.

Nyhlin H, Bang C, Elsborg L, et al. 2004. A double-blind, placebocontrolled, randomized study to evaluate the efficacy, safety and tolerability of tegaserod in patients with irritable bowel syndrome. Scandinavian Journal of Gastroenterology, 39:119-26.

Prather CM, Camilleri M, Zinsmeister AR, et al. 2000. Tegaserod accelerates orocecal transit in patients with constipation-predominant irritable bowel syndrome. Gastroenterology, 118:463-8.

Reilly MC, Barghout V, McBurney CR, et al. 2005. Effect of tegaserod on work and daily activity in irritable bowel syndrome with constipation. Alimentary Pharmacology and Therapeutics, 22:373-80.

Sandler RS, Everhart JE, Donowitz M, et al. 2002. The burden of selected digestive diseases in the United States. Gastroenterology, 122:1500-11.

Schoenfeld P. 2004. Review article: the safety profile of tegaserod. Alimentary Pharmacology and Therapeutics, 20(Suppl 7):25-30.

Smith DG, Barghout V, Kahler KH. 2005. Tegaserod treatment for IBS: a model of indirect costs. American Journal of Managed Care, 11(1 Suppl):S43-50.

Tabas G, Beaves M, Wang J, et al. 2004. Paroxetine to treat irritable bowel syndrome not responding to high-fiber diet: a double-blind, placebocontrolled trial. American Journal of Gastroenterology. 99:914-20.

Tack J, Broekaert D, Fischler B, et al. 2006. A controlled cross-over study of the selective serotonin reuptake inhibitor citalopram in irritable bowel syndrome. Gut, 55:1095-103.

Talley NJ, Gabriel SE, Harmsen WS, et al. 1995. Medical costs in community subjects with irritable bowel syndrome. Gastroenterology, 109:1736-41.

Talley NJ. 2001. Serotonergic neuroenteric modulators. Lancet, 358:2061-8.

Talley NJ. 2003. Pharmacologic therapy for the irritable bowel syndrome. American Journal of Gastroenterology, 98:750-8.

Talley NJ. 2004. Antidepressants in IBS: are we deluding ourselves? Am J Gastroenterol, 99:921.

Traynor K. 2004. Alosetron use drops dramatically with risk management. American Journal of Health-System Pharmacy, 61:1210-12.

Vahedi H, Merat S, Rashidioon A, et al. 2005. The effect of fluoxetine in patients with pain and constipation-predominant irritable bowel syndrome: a double-blind randomized-controlled study. Alimentary Pharmacology and Therapeutics, 22:381-5.

Viramontes BE, Camilleri M, McKinzie S, et al. 2001. Gender-related differences in slowing colonic transit by a 5 -HT3 antagonist in subjects with diarrhea-predominant irritable bowel syndrome. American Journal of Gastroenterology, 96:2671-6.

Whitehead WE, Burnett CK, Cook EW, et al. 1996. Impact of irritable bowel syndrome on quality of life. Dig Dis Sci, 41:2248-53.

Wolfe SG, Chey WY, Washington MK, et al. 2001. Tolerability and safety of alosetron during long-term administration in female and male irritable bowel syndrome patients. American Journal of Gastroenterology, 96:803-11. 